\title{
APPROXIMATE DESIGN METHOD OF MRAC WITH NN FOR PNEUMATIC SERVO SYSTEM
}

\author{
Tadashi MATSUSHITA, Jinhua LI, Yoshiki MIZUKAMI and Kanya TANAKA \\ Department of Electrical and Electronic Engineering, Faculty of Engineering \\ Yamaguchi University \\ 2-16-1, Tokiwadai, Ube-city, Yamaguchi, 755-8611 Japan \\ (E-mail: matsushita@ctrl.eee.yamaguchi-u.ac.jp)
}

\begin{abstract}
Pneumatic servo systems are used widely in industrial field. However, the dynamic characteristics often change with load mass and there are non-linear elements caused by air compressibility, various frictions and so on. Therefore, it is difficult for conventional linear control methods to accomplish satisfactory control performance. In addition, there is a problem that discrete-time models of pneumatic servo system have possibilities to become non-minimum phase by difference of the sampling period, change in load mass, and so on.

In this paper, for these problems, we propose a design scheme which combines a Model Reference Adaptive Control (MRAC) using delta-operator and a Neural Network (NN). In this design, even if the discrete-time model is non-minimum phase, it can be constructed by regarding approximate model of the plant expressed by delta-operator as minimum-phase model, and NN compensates for modeling error and the non-linearity of pneumatic servo systems.
\end{abstract}

\section{KEY WORDS}

Model Reference Adaptive Control, Neural Network, Delta-Operator

\section{INTRODUCTION}

Recently, the eco-friendly actuators become important for the protection of environment increasingly. In the pneumatic system, since a transfer medium is air, there are not risks of ignition, explosion or pollution like the hydraulic system. So it is thought that it will be seriously taken further in respect of the protection of environment from now on. Especially, the pneumatic servo system is excellent in economical efficiency, safety, etc., and is utilized in many fields of the industrial world. However, the dynamic characteristics often change with load mass and there are non-linear elements caused by air compressibility, various frictions and so on. Therefore, it is difficult to accomplish satisfactory control performance by using PID or conventional methods. In a Model Reference Adaptive Control (MRAC), according to the change of dynamic characteristics, the parameters of a controller is automatically adjusted with the dynamic characteristics in the on-line procedure, and MRAC always keeps the good performance. The construction of an adaptive control system is so much complex that it is usually realized by using a digital-computer to discrete-time model of a plant. But 
discrete-time model of pneumatic servo system have possibilities to become non-minimum phase according to the sampling period, the load mass, and so on. Thus, by MRAC, when the plant becomes the non-minimum phase, MRAC can not be adapted because the control input may diverge. Then MRAC using delta-operator as construction which can be used for the plant of the non-minimum phase system is proposed [1-3]. In the construction using this delta-operator, by making a sampling period minimum, it can be regarded that the obtained approximate model is the minimum phase system. However, existent pneumatic servo systems contain non-linear element, so it is difficult to accomplish the satisfactory control performance by applying the conventional MRAC using delta-operator. To overcome this problem, we propose a design scheme of MRAC using delta-operator with a Neural Network (NN) in this paper [4]. In this design, NN compensates for modeling error and the non-linearity of pneumatic servo systems. Therefore, a good control performance can be attained by using $\mathrm{NN}$ together.

\section{THE PNEUMATIC SERVO SYSTEM}

Fig.1 shows a schematic diagram of the pneumatic servo system considered in this paper. The air cylinder is a both-rods type with the inside diameters of the cylinder of $55 \mathrm{~mm}$, a stroke of $170 \mathrm{~mm}$, and rod-diameters of $22 \mathrm{~mm}$. The piston and rod are sealed with the labyrinth packing and the air bearings, respectively. The cylinder is placed horizontally and is constructed so that the load can be moved in the horizontal direction. The control valves are three-directional electro-pneumatic proportional pressures ones. The magnescale sensor is used for detection of the position of the load. The signal from the magnescale sensor is inputted into the computer through the counter. The control signal is calculated in the computer and transmitted to the control values through the D/A converters and the power amplifiers. The cylinder is moved by the air pressure adjusted with the control values.

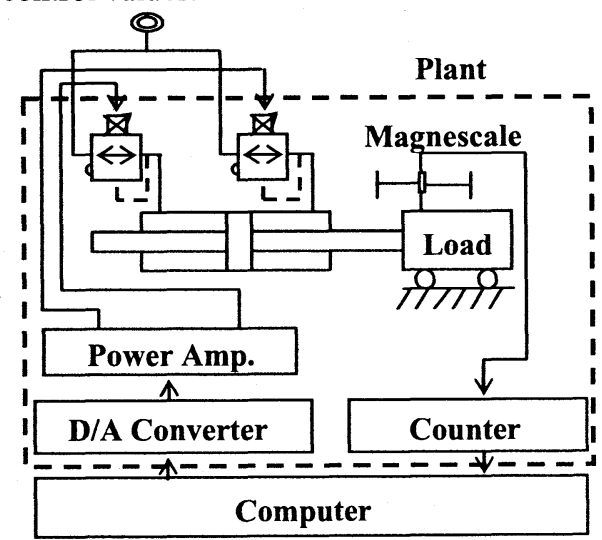

Fig.1 Schematic diagram of the pneumatic servo system.

\section{MODELING OF THE PLANT}

The portion surrounded with dashed line in Fig. 1 is considered as the plant in this paper. From our preliminary experiment, the time-delay of this plant can be regarded as $0.04 \mathrm{sec}$.

The linearized discrete-time model is described as

$$
\begin{aligned}
& y(k)=\frac{B\left(z^{-1}\right)}{A\left(z^{-1}\right)} u(k-d), \\
& \left.\begin{array}{l}
A\left(z^{-1}\right)=1+a_{1} z^{-1}+a_{2} z^{-2}+a_{3} z^{-3}, \\
B\left(z^{-1}\right)=b_{0} z^{-1}+b_{1} z^{-2}+b_{2} z^{-3}, \quad d=L / T,
\end{array}\right\}
\end{aligned}
$$

\section{where}

$u(k)$ and $y(k)$ express the control signal and the output of plant, respectively. We should note that this discrete-time model may become the non-minimum phase system. In such a plant, MRAC can not be constituted. Therefore, we adopt the delta-operator described as

$$
\delta=\frac{z-1}{T} \text {. }
$$

By using the delta-operator, Eq.(1) can be rewritten as

$$
y(k)=\frac{D(\delta)}{C(\delta)} u(k-d)
$$

where

$$
\begin{aligned}
& C(\delta)=\delta^{3}+\alpha_{1} \delta^{2}+\alpha_{2} \delta, \\
& \left.D(\delta)=\beta_{0} \delta^{2}+\beta_{1} \delta+\beta_{2},\right\} \\
& d=0.04 / T \text {, } \\
& \alpha_{1}=\frac{3+a_{1}}{T}, \quad \alpha_{2}=\frac{3+2 a_{1}+a_{2}}{T^{2}}, \\
& \beta_{0}=\frac{b_{0}}{T}, \quad \beta_{1}=\frac{2 b_{0}+b_{1}}{T^{2}}, \quad \beta_{2}=\frac{b_{0}+b_{1}+b_{2}}{T^{3}} .
\end{aligned}
$$

In Eq.(6), when $T \rightarrow 0$, the plant parameters, $\beta_{0}$ and $\beta_{1}$ converge to 0 owing to the characteristics of $b_{0}$ and $b_{1}$. Then, if we choose a sampling period with $0.02 \mathrm{sec}$., $\beta_{0}$ and $\beta_{1}$ are regarded as 0 . Therefore, the approximate model is described below

$$
y(k)=\frac{\beta_{2}}{C(\delta)} u(k-2) \text {. }
$$

In the next section, our design scheme of MRAC for the approximate model is described.

\section{DESIGN SCHEME OF MRAC}

The following equation is considered as the reference model for the approximate model shown by Eq.(7).

$$
y_{M}(k)=\frac{\beta_{M}}{A_{M}(\delta)} r(k-2),
$$

where 


$$
A_{M}(\delta)=\delta^{3}+\alpha_{M 1} \delta^{2}+\alpha_{M 2} \delta+\alpha_{M 3}
$$

In Eq.(8), $r(k)$ is a bounded reference input and $A_{M}(\delta)$ is a Hurwits polynomial. The framework of MRAC gives control signal adaptationally so that the output of plant $y(k)$ may follow that of reference model $y_{M}(k)$. Then, the construction of MRAC which attains this control purpose is described. The following Hurwits polynomial $F(\delta), \bar{F}(\delta)$ and $H(\delta)$ are introduced.

$$
\left.\begin{array}{rl}
F(\delta) & =\bar{F}(\delta)(\delta+f)^{2} \\
& =\delta^{4}+\sum_{i=1}^{4} f_{i} \delta^{4-i}, \\
\bar{F}(\delta) & =\delta^{2}+\bar{f}_{1} \delta+\bar{f}_{2}, \quad f=\frac{1}{T}, \\
H(\delta) & =\delta^{3}+h_{1} \delta^{2}+h_{2} \delta+h_{3} .
\end{array}\right\}
$$

It notes that the polynomials $R(\delta), S(\delta)$ which satisfy the next relation exist for $C(\delta)$ of Eq.(7).

$$
F(\delta) H(\delta)=C(\delta) R(\delta)+S(\delta)
$$

where

$$
\left.\begin{array}{l}
R(\delta)=\delta^{4}+r_{1} \delta^{3}+r_{2} \delta^{2}+r_{3} \delta+r_{4}, \\
S(\delta)=s_{0} \delta^{2}+s_{1} \delta+s_{2} .
\end{array}\right\}
$$

Multiplying both sides in Eq.(11) by $y(k+2)$ and using Eq.(7) to it, we have that

$$
\begin{aligned}
y(k+2) & =\frac{1}{H(\delta)}\left\{\frac{\beta_{2} R(\delta)}{F(\delta)} u(k)+\frac{S(\delta)(1+T \delta)^{2}}{F(\delta)} y(k)\right\} \\
& =\frac{1}{H(\delta)} \phi^{T} \xi(k),
\end{aligned}
$$

where

$$
\begin{aligned}
& \phi=\left[\phi_{1}, \cdots \phi_{8}\right]^{T}, \\
& \phi_{1}=\beta_{2}, \quad \phi_{i+1}=\beta_{2}\left(r_{i}-f_{i}\right), i=1 \sim 4, \\
& \left.\phi_{6}=\frac{s_{0}}{f^{2}}, \quad \phi_{6+i}=\frac{s_{i}-s_{0} \bar{f}_{i}}{f^{2}} ; i=1,2, \quad\right\} \\
& \xi(k)=\left[\xi_{1}(k), \cdots, \xi_{8}(k)\right]^{T}, \\
& \xi_{1}(k)=u(k), \xi_{i+1}(k)=\frac{\delta^{4-i}}{F(\delta)} u(k) ; i=1 \sim 4, \\
& \xi_{6}(k)=y(k), \quad \xi_{6+i}(k)=\frac{\delta^{2-i}}{\bar{F}(k)} y(k), i=1,2 .
\end{aligned}
$$

In Eq.(13), a control input is synthesized as follows, by replacing $y(k)$ and $\phi$ with $y_{M}(k)$ and the variable parameter $\hat{\phi}(k)=\left[\hat{\phi}_{1}(k), \cdots \hat{\phi}_{8}(k)\right]^{T}$, respectively.

$$
\begin{aligned}
& \hat{\phi}^{T}(k) \xi(k)=y_{M H}(k+2), \\
& u(k)=\frac{y_{M H}(k+2)-\sum_{i=2}^{8} \hat{\phi}_{i}(k) \xi_{i}(k)}{\hat{\phi}_{1}(k)},
\end{aligned}
$$

where

$$
y_{M H}(k)=H(\delta) y_{M}(k)
$$

In practice, since parameters of the plant are unknown, it is required to identify those unknown parameters and to decide the plant parameters by using the estimated parameters. Then, the identification method of the plant parameters is shown below. Eq.(1) is rewritten as

$$
y(k)=\theta^{T} \zeta(k),
$$

where

$$
\begin{aligned}
& \theta=\left[a_{1}, a_{2}, a_{3}, b_{0}, b_{1}, b_{2}\right]^{T}, \\
& \zeta(k)=\left[\zeta_{1}(k), \cdots, \zeta_{6}(k)\right]^{T}, \\
& \zeta(k)=u(k-2-i) \zeta_{3+i}(k)=y(k-i) j=1 \sim 3 .
\end{aligned}
$$

Corresponding to Eq.(19), the identification model is considered as follows.

$$
\hat{y}(k)=\hat{\theta}^{T}(k) \zeta(k)
$$

where

$$
\hat{\theta}(k)=\left[\widehat{a}_{1}(k), \hat{a}_{2}(k), \hat{a}_{3}(k), \hat{b}_{0}(k), \hat{b}_{1}(k), \widehat{b}_{2}(k)\right] \text {. }
$$

Define the identification error as

$$
\varepsilon(k)=\hat{y}(k)-y(k)=\zeta^{T}(k) \hat{\theta}(k)-y(k)
$$

then, the estimated parameters $\hat{\theta}(k)$ are identified by using the constant trace algorithm as follows.

$$
\begin{aligned}
& \hat{\theta}(k)=\hat{\theta}(k-1)-\frac{\Gamma(k-1) \zeta(k) \varepsilon(k)}{1+\zeta^{T}(k) \Gamma(k-1) \zeta(k)}, \\
& \hat{\Gamma}(k-1)=\Gamma(k-1)-\frac{\Gamma(k-1) \zeta(k) \zeta^{T}(k) \Gamma(k-1)}{1+\zeta^{T}(k) \Gamma(k-1) \zeta(k)}, \\
& \lambda(k)=\frac{\operatorname{tr} \hat{\Gamma}(k-1)}{\operatorname{tr} \hat{\Gamma}(0)}, \\
& \Gamma(k)=\frac{\hat{\Gamma}(k-1)}{\lambda(k)} .
\end{aligned}
$$

Considering the estimated parameters $\hat{\theta}(k)$ obtained from Eq.(26) as the true values, control parameters are calculated as follows.

$$
\left.\begin{array}{l}
\hat{\phi}_{1}(k)=\hat{\beta}_{2}(k), \\
\hat{\phi}_{i+1}(k)=\hat{\beta}_{2}(k)\left(\hat{r}_{i}(k)-f_{i}\right) ; i=1 \sim 4, \\
\hat{\phi}_{6}(k)=\frac{\hat{s}_{0}(k)}{f^{2}}, \\
\hat{\phi}_{6+i}(k)=\frac{\hat{s}_{i}(k)-\hat{s}_{0}(k) \bar{f}_{i}}{f^{2}} ; i=1,2, \\
\hat{\alpha}_{1}(k)=\frac{3+\hat{a}_{1}(k)}{T}, \quad \hat{\alpha}_{2}(k)=\frac{3+2 \hat{a}_{1}(k)+\hat{a}_{2}(k)}{T^{2}}, \\
\hat{\beta}_{0}(k)=\frac{\hat{b}_{0}(k)}{T}, \quad \hat{\beta}_{1}(k)=\frac{2 \hat{b}_{0}(k)+\hat{b}_{1}(k)}{T^{2}}, \\
\hat{\beta}_{2}(k)=\frac{\hat{b}_{0}(k)+\hat{b}_{1}(k)+\hat{b}_{2}(k)}{T^{3}},
\end{array}\right\}
$$




$$
\left.\begin{array}{rl}
\hat{r}_{1}(k)=f_{1}+h_{1}-\hat{\alpha}_{1}(k), \\
\hat{r}_{2}(k)=f_{2}+f_{1} h_{1}+h_{2}-\hat{\alpha}_{2}(k)-\hat{\alpha}_{1}(k) \hat{r}_{1}(k), \\
\hat{r}_{3}(k)=f_{3}+f_{2} h_{1}+f_{1} h_{2}+h_{3}-\hat{\alpha}_{2}(k) \hat{r}_{1}(k) \\
\quad-\hat{\alpha}_{1}(k) \hat{r}_{2}(k), \\
\hat{r}_{4}(k)=f_{4}+f_{3} h_{1}+f_{2} h_{2}+f_{1} h_{3}-\hat{\alpha}_{2}(k) \hat{r}_{2}(k) \\
\quad-\hat{\alpha}_{1}(k) \hat{r}_{3}(k), \\
\hat{s}_{0}(k)=f_{4} h_{1}+f_{3} h_{2}+f_{2} h_{3}-\hat{\alpha}_{2}(k) \hat{r}_{3}(k) \\
\quad-\hat{\alpha}_{1}(k) \hat{r}_{4}(k), \\
\hat{s}_{1}(k)=f_{4} h_{2}+f_{3} h_{3}-\hat{\alpha}_{2}(k) \hat{r}_{4}(k), \\
s_{2}=f_{4} h_{3} .
\end{array}\right\}
$$

\section{DESIGN SCHEME OF MRAC WITH NN}

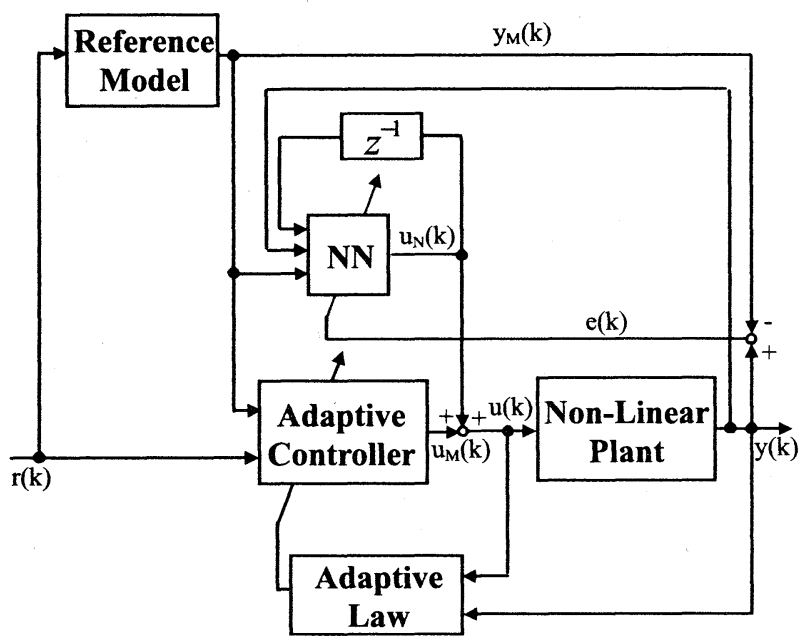

Fig.2 Schematic diagram of MRAC with NN.

In practice, since the pneumatic servo systems contain non-linear elements caused by compressibility of the air, various frictions and so on, it is difficult to accomplish satisfactory control performance. Thus, we propose approximate design method of MRAC with NN that constitutes the controller of MRAC and NN in parallel illustrated in Fig.2. In this design, NN trains to compensate for modeling error and the non-linearity of pneumatic servo systems. The control input $u(k)$ is synthesized as the sum of the output of the adaptive controller, $u_{M}(k)$ and the output of NN, $u_{N}(k)$.

$$
u(k)=u_{M}(k)+u_{N}(k) \text {. }
$$

We use the architecture of the 3-layers type NN. The input-layer consists of 8 units and their output signals are described as

$$
\left\{x_{i}(k)\right\}=\left\{y_{M H}(k+2), \varsigma_{2}(k), \varsigma_{3}(k),\right.
$$

$$
\left.\varsigma_{4}(k), \varsigma_{5}(k), \varsigma_{6}(k), \varsigma_{7}(k), \varsigma_{8}(k)\right\}
$$

where

$$
\left.\begin{array}{l}
\varsigma_{i+1}(k)=\frac{\delta^{4-i}}{F(\delta)} u_{N}(k) ; i=1 \sim 4, \\
\varsigma_{6}(k)=y(k), \quad \varsigma_{6+i}(k)=\frac{\delta^{2-i}}{\bar{F}(k)} y(k), i=1,2 .
\end{array}\right\}
$$

The hidden-layer consists of 20 units. The output signals in the hidden and output-layer are, respectively, described as

$$
\begin{aligned}
& h_{j}(k)=f\left(\sum_{i=1}^{8} x_{i}(k) w_{i j}^{(1)}(k)\right), \\
& u_{N}(k)=\sum_{j=1}^{20} w_{j}^{(2)}(k) h_{j}(k),
\end{aligned}
$$

where

$$
f(x)=\frac{1}{1+\exp (-x)} \text {. }
$$

Define the output-error as

$$
e(k)=y(k)-y_{M}(k) \text {, }
$$

Using the back-propagation (BP) algorithm of $\mathrm{NN}$, the weights $w_{i j}^{(1)}(k), w_{j}^{(2)}(k)(i=1 \sim 8, j=1 \sim 20)$ are updated so as to decrease the square of the output-error. That is

$$
\begin{aligned}
w_{i j}^{(1)}(k+1)= & w_{i j}^{(1)}(k)-\eta e(k) \frac{\partial y(k)}{\partial u(k-3)} w_{j}^{(2)}(k-3) \\
& \times h_{j}(k-3)\left(1-h_{j}(k-3)\right) x_{i}(k-3), \\
w_{j}^{(2)}(k+1)= & w_{j}^{(2)}(k)-\eta e(k) \frac{\partial y(k)}{\partial u(k-3)} h_{j}(k-3),
\end{aligned}
$$

where, $\eta(>0)$ is the leaning rate of the algorithm. The value of Jacobian, $\partial y(k) / \partial u(k-3)$, is given by the following equation,

$$
\frac{\partial y(k)}{\partial u(k-3)}=\hat{b}_{0}(k)
$$

Fig.3 shows the architecture of the 3-layers type NN used in our design scheme.

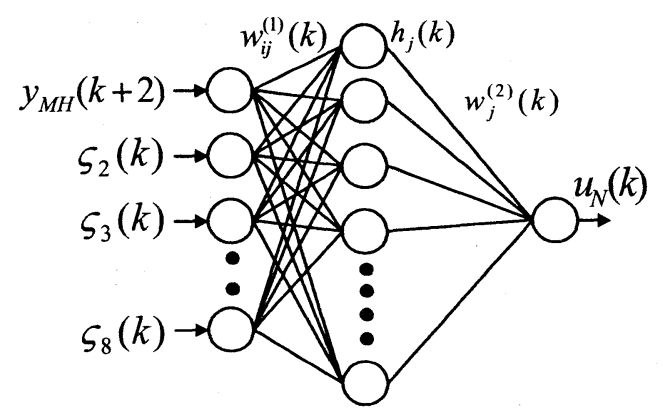

Fig.3 Architecture of NN. 


\section{EXPERIMENTAL RESULTS}

This section shows the experimental results which were obtained by using the existent pneumatic servo system to confirm the effectiveness of proposed design scheme.

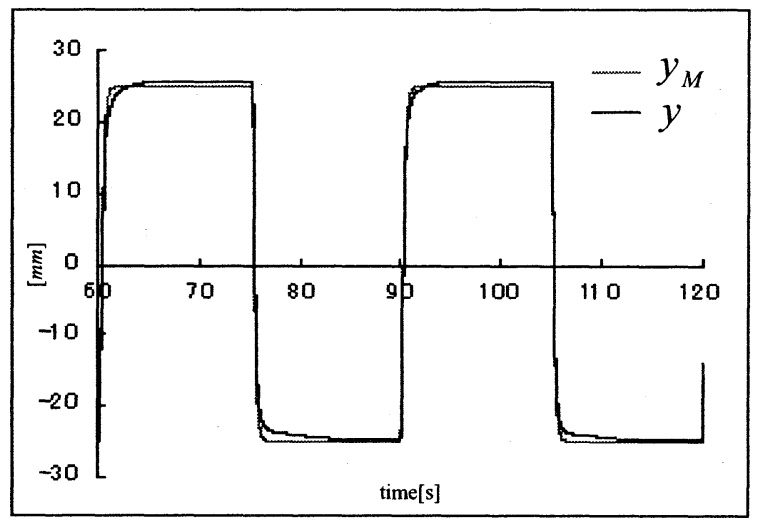

(a) output of the reference model and plant.

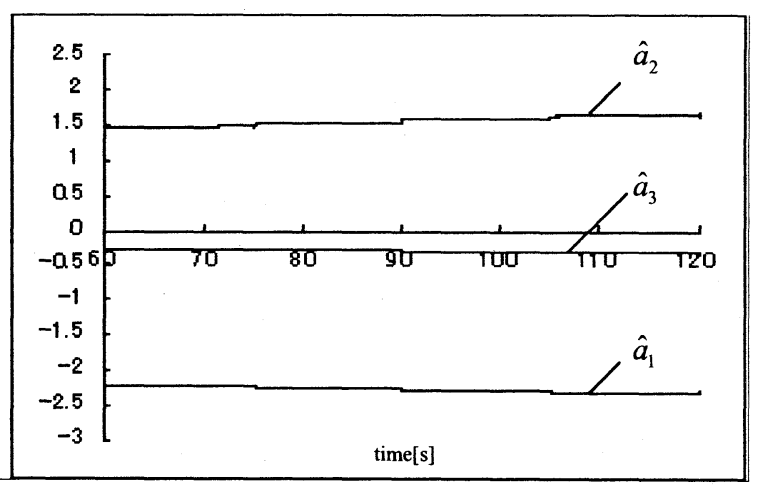

(b) estimated parameters $\hat{a}_{i}(1 \leq i \leq 3)$.

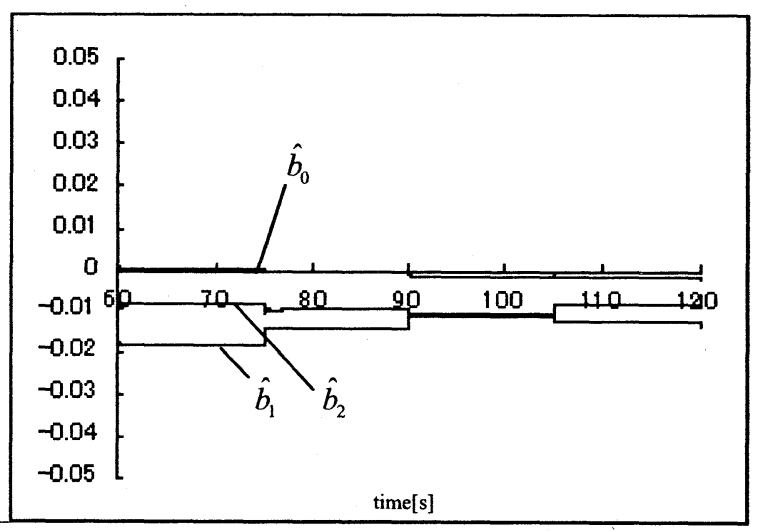

(c) estimated parameters $\hat{b}_{i} \quad(0 \leq i \leq 2)$.

Fig.4 Transient responses of MRAC with NN.
Parameters used in the control algorithm are chosen as $\alpha_{M 1}=30.30, \alpha_{M 2}=270.09, \alpha_{M 3}=710.87, \beta_{M}=710.87$, $\bar{f}_{1}=100, \bar{f}_{2}=2500, f=50, \Gamma(0)=10 I$, $h_{1}=30.30, h_{2}=270.09, h_{3}=710.87$.

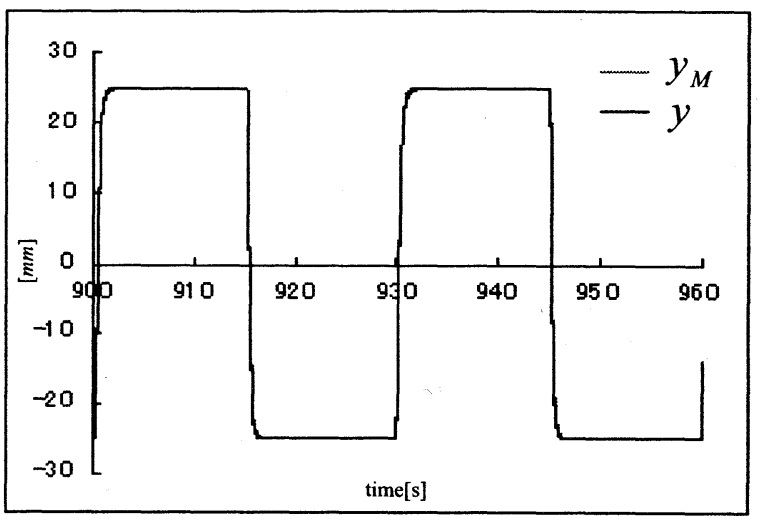

(a) output of the reference model and plant.

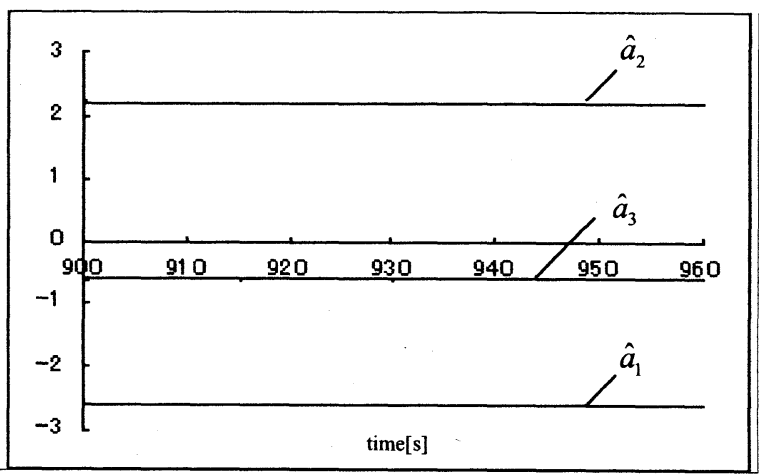

(b) estimated parameters $\hat{a}_{i}(1 \leq i \leq 3)$.

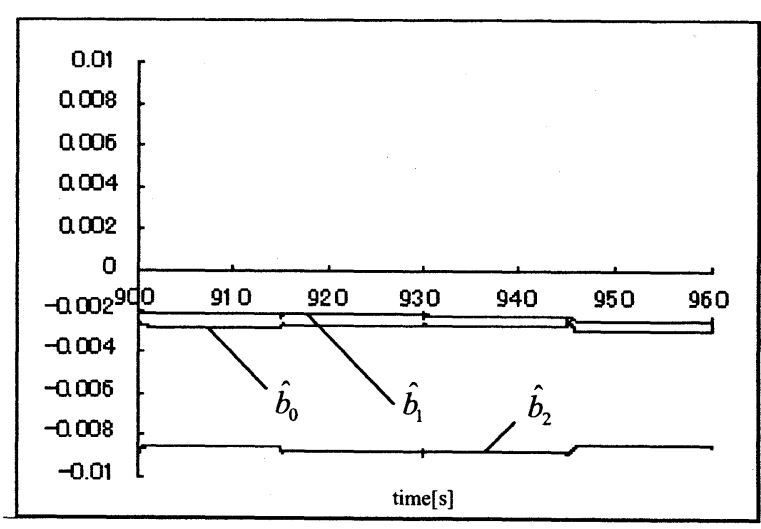

(c) estimated parameters $\hat{b}_{i} \quad(0 \leq i \leq 2)$.

Fig.5 Stationary responses of MRAC with NN. 
The initial values of the controller parameters were decided as

$$
\begin{aligned}
& \hat{a}_{1}(0)=-2.3, \hat{a}_{2}(0)=1.9, \hat{a}_{3}(0)=-0.5, \\
& \hat{b}_{0}(0)=0.1, \hat{b}_{1}(0)=0.1, \hat{b}_{2}(0)=-0.1 .
\end{aligned}
$$

The leaning rate $\eta$ is chosen as 0.01 and the initial values of the weights, $w_{i j}^{(1)}(0)$ and $w_{j}^{(2)}(0)$, are given with the random numbers at the range of [-0.001:0.0001]. We adopted a rectangle wave as the reference input $r(k)$, which has the amplitude of $\pm 25 \mathrm{~mm}$ and the period of $30 \mathrm{sec}$.

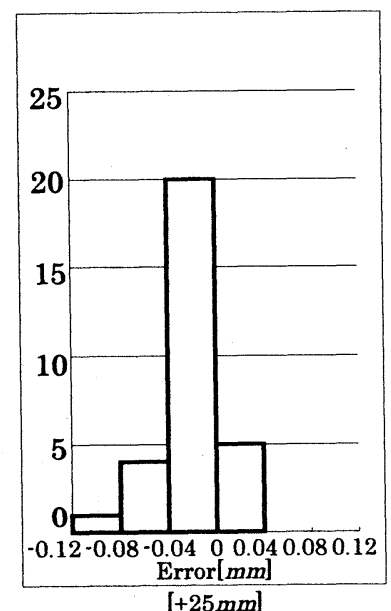

Fig.6 The position accuracy of the conventional MRAC.

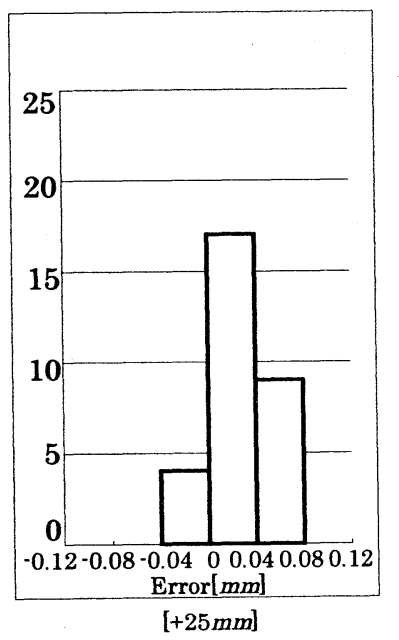

Fig. 7 The position accuracy of MRAC with NN.

Fig.4 and Fig.5 show the transient and stationary responses of MRAC with NN. Fig.(a) shows the reference model output $y_{M}(k)$ and the plant output $y(k)$. Fig.(b) and Fig.(c) show the estimated controller parameters $\hat{\theta}(k)$, respectively. From these results, Fig.4 shows that the controller parameters were not constant and it is difficult for the output of plant accurately tracks to that of the reference model in the transient response. However, in the stationary response, the controller parameters converged to the constant values and the output of plant tracked to that of the reference model as shown in Fig.5.

Fig.6 and Fig. 7 are the histograms which show 30 times iterative positioning experimental results of the measured at $12 \mathrm{sec}$. after change of the sign of the reference input $r(k)$. Fig.6 shows the experimental results of the conventional design scheme, i.e., MRAC without NN. It can be seen that the positioning errors are within $\pm 0.12 \mathrm{~mm}$. On the other hand, Fig. 7 shows ones of the proposed design scheme, i.e., MRAC with NN. It can be seen that positioning errors are within $\pm 0.08 \mathrm{~mm}$, it shows the superior positioning accuracy. These results clarify that $\mathrm{NN}$ compensated for modeling error and the non-linearity of pneumatic servo systems.

\section{CONCLUSION}

This paper presented the approximated design method of MRAC with NN for the pneumatic servo system. The effectiveness of this design scheme has been confirmed by the experiments using the existent pneumatic servo system.

\section{REFERENCES}

[1] K.Tanaka, A.Shimizu, S.Shibata, M.Senoo, and M.Kurrigami, "MRAC Using $\delta$ - operator for Electro-Pneumatic Servo Systems", T.IEE Japan, 1995, Vol.115-D, No.5, pp.605-611

[2] K.Araki, Y.Yamakawa, H.Waji, and A.Yumuro, " $\delta$-Operator Model Reference Adaptive Control of a Pneumatic Servo", Fluid Power System, 1994, 23-3, pp.91-98

[3] M.Kurigami, K.Tanaka, and A.Shimizu, "Multirate MRACS Using $\delta$-Operator for Electro-Pneumatic Servo Systems with Additive External Forces", T.IEE Japan, 1997, Vol.177-D, No.9, pp.1069-1076

[4] J.Tsuji, H.Ohmori and A.Sano, "Adaptive Control Incorporating Neural Network", Trans. on Society of Instrument Control Engineers, 1994, Vol.30, No.3, pp.295-302 